\title{
Necrólisis epidérmica tóxica y lupus eritematoso sistémico
}

\author{
Toxic epidermal necrolysis and systemic lupus erythematosus
}

\author{
Catalina Santa-Vélez' ${ }^{1}$ CvLAC, Nathalie Morales', Juliana Calle', Javier D. Márquez², Luis \\ Fernando Pinto-Peñaranda2 ${ }^{2 v L A C}$, Carlos J. Velásquez ${ }^{2}$
}

\begin{abstract}
Fecha correspondencia:
Recibido: octubre 21 de 2013.

Revisado: octubre 31 de 2014.

Aceptado: enero 30 de 2015.
\end{abstract}

Forma de citar:

Santa-Vélez C, Morales N, Calle J, Márquez JD, Pinto-Peñaranda

LF, Velásquez CJ. Necrólisis

epidérmica tóxica y lupus

eritematoso sistémico. Rev CES

Med 2018; 32(1): 67-73.

\author{
Open access \\ (C) Derecho de autor \\ Licencia creative commons \\ Ética de publicaciones \\ Revisión por pares \\ Gestión por Open Journal System \\ DOI: http://dx.doi.org/10.21615/ \\ cesmedicina.32.1.8 \\ ISSN 0120-8705 \\ e-ISSN 2215-9177
}

Sobre los autores:

1. Dermatóloga.

2. Reumatólogo HPTU.

3. Reumatólogo Clínica Bolivariana.

Comparte

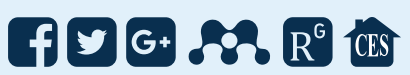

\section{Resumen}

El lupus eritematoso sistémico con manifestación tipo necrólisis epidérmica tóxica es una entidad descrita recientemente y cada vez aparecen más reportes en la literatura. Se describe el caso de una paciente de 15 años con lupus eritematoso sistémico quien presentó una necrólisis epidérmica tóxica extensa, cuyas lesiones iniciales eran tipo eritema multiforme. Se discute el caso a la luz de la literatura actual sobre esta nueva entidad.

Palabras clave: Necrolisis epidérmica tóxica; Eritema multiforme; Síndrome de Stevens Johnson; Lupus eritematoso sistémico; Lupus eritematoso cutáneo.

\begin{abstract}
Toxic epidermal necrolysis (TEN)-like systemic lupus erythematosus is a recently described entity and more cases are being published in the literature today. We describe the case of a 15-year old patient with systemic lupus erythematosus who developed TEN that initially started with erythema multiforme (EM)-like lesiones. We discuss this case according to the published literature on this new entity.
\end{abstract}

Keywords: Toxic epidermal necrolysis; Erythema multiforme; Stevens-Johnson syndrome; Systemic lupus erythematosus; Cutaneous lupus erythematosus

\section{Caso clínico}

Se trataba de una niña de 15 años, residente en el departamento de Antioquia, con un cuadro clínico de tres meses de evolución que consistía en edema progresivo de cara y los miembros inferiores, junto a disnea de medianos esfuerzos y artralgias con artritis de ambas manos. Lo anterior asociado a un cuadro de 15 días de evolución de un rash morbiliforme en áreas fotoexpuestas, muy pruriginoso, que posteriormente se generalizó. La paciente consultó a un hospital de segundo nivel de atención, en donde le encontraron, adicionalmente, cifras elevadas de presión arterial.

La menor fue hospitalizada por el servicio de medicina Interna y se le inició manejo con esteroides tópicos y antihistamínicos y le fueron solicitados exámenes paraclínicos, que reportaron: $\mathrm{Hb}$ 7,2, leucocitos de 5800 (81\% de neutrófilos y $12 \%$ linfocitos), anti-SM y anti-ADN positivos, anticuerpos antinucleares ( $A N A^{\prime} S$ ) reactivos 1:320 con patrón moteado, anti-Ro y anti-La negativos, velocidad de sedimentación globular 60, creatinina 2,05, 
La paciente presentó deterioro del estado general, persistencia de las cifras de presión altas, con aparición de lesiones en diana en extremidades y tronco y ampollas tensas de forma generalizada, características sugestivas de un síndrome de Rowell (lesiones de eritema multiforme en el contexto de un lupus sistémico)
BUN 42; citoquímico de orina con 150 de proteinuria y proteínas en orina 24 horas de $111 \mathrm{mg} / \mathrm{dL}$. Por lo anterior, fue remitida a un hospital de tercer nivel de complejidad, con diagnóstico de lupus eritematoso sistémico con compromiso renal y cutáneo.

A su ingreso se encontró una paciente pálida, con leve dificultad respiratoria y en la auscultación un soplo sistólico grado III/IV en el foco mitral. Por esta razón se le ordenó ecocardiografía, en la que se evidenció insuficiencia mitral e hipertensión pulmonar moderadas y se ordenó interconsulta por el servicio de dermatología para el diagnóstico y manejo de las lesiones en piel.

Al momento de la evaluación presentaba erosiones en mucosas y vesículas dolorosas en paladar duro. En piel, se le encontraron placas eritemato-violáceas, edematosas en región malar, que respetaban los surcos nasogenianos, pero con extensión hasta el tórax anterior.

Basados en la historia clínica, los hallazgos al examen físico y los paraclínicos se realizó diagnóstico de lupus eritematoso sistémico con compromiso hematológico, renal, cutáneo y posiblemente cardiovascular, por lo que le iniciaron pulsos de metilprednisolona, así como losartan y cloroquina y según evolución, se planteó la posibilidad de iniciar bolos de ciclofosfamida.

Al día siguiente la paciente presentó deterioro del estado general, persistencia de las cifras de presión altas, con aparición de lesiones en diana en extremidades y tronco y ampollas tensas de forma generalizada, características sugestivas de un síndrome de Rowell (lesiones de eritema multiforme en el contexto de un lupus sistémico) (figuras 1 y 2).

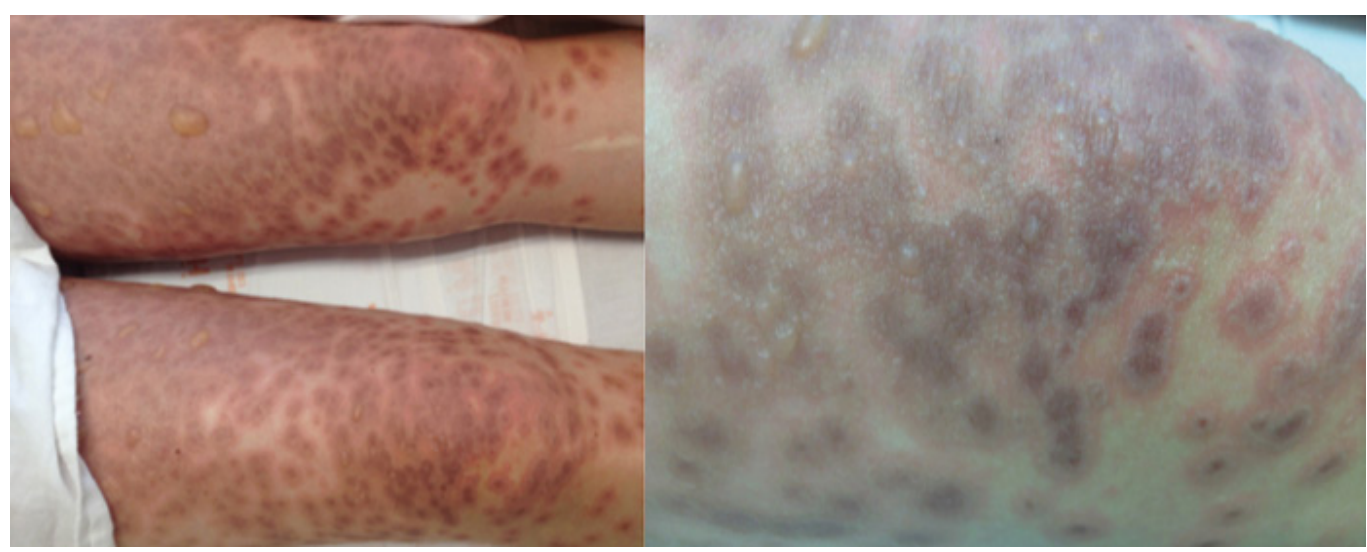

Figuras 1 y 2. Lesiones sugestivas de síndrome de Rowell (lesiones de eritema multiforme en lupus sistémico

Por tal razón se inició remisión a un cuarto nivel de complejidad para manejo conjunto por reumatología y dermatología. Un día mas tarde, en espera del proceso de remisión, la paciente presentó aumento de las lesiones en piel, con esfacelación espontánea y signo de Nikolsky, además de marcado compromiso de mucosas oral, genital y ocular (figura 3 ).

Debido a la severidad del cuadro, con lesiones de necrólisis epidérmica tóxica en el contexto de un lupus sistémico en franca actividad, el compromiso renal y riesgo de muerte se iniciaron bolos de ciclofosfamida y se trasladó a un cuarto nivel de complejidad, donde murió por falla renal. 


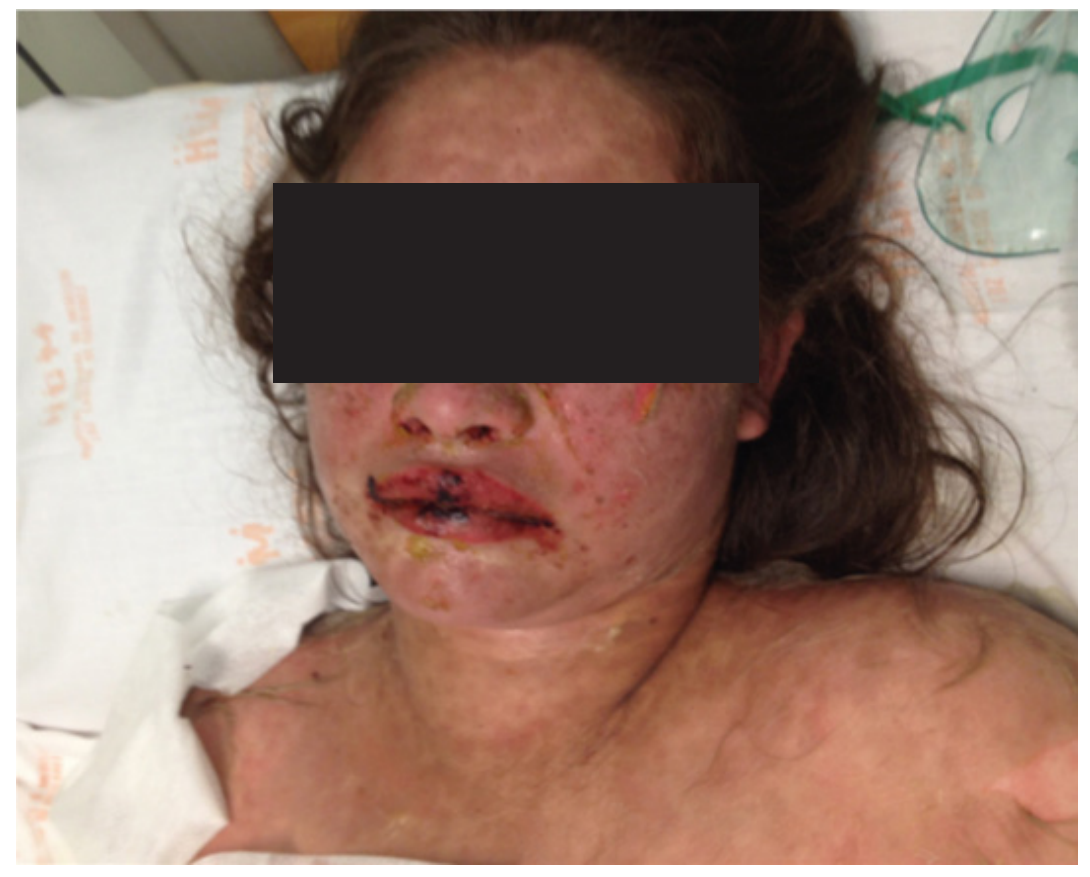

Figura 3. Esfacelación cutánea por necrolisis epidérmica tóxica

\section{Discusión}

El lupus eritematoso sistémico se ha asociado a múltiples manifestaciones vesicoampollosas (1,2). Estas pueden ser manifestaciones inespecíficas, o sea, entidades asociadas a auto-anticuerpos que no ocurren como extensión de la dermatitis de interface característica de la enfermedad dermatológica específica del lupus, como el lupus vesicoampolloso ( $\underline{3}-\underline{7})$ o las enfermedades ampollosas primarias (dermatitis herpetiforme (요 o epidermolisis ampollosa adquirida) $(\underline{1}, \underline{2}, \underline{9})$.

O por el contrario, pueden ser manifestaciones vesicoampollosas específicas del lu-

La necrólisis epidérmica tóxica (NET) asociada al lupus eritematoso cutáneo agudo o subagudo, que se caracteriza por lesiones tipo NET en regiones típicas de lupus cutáneo agudo o subagudo con tendencia a que las lesiones estén en áreas fotodistribuidas. pus, que ocurren como resultado de la inflamación agresiva con daño de la membrana basal, debido a extensión dramática de la dermatitis de interface característica de la enfermedad dermatológica por lupus eritematoso $(\underline{1}, \underline{2}, \underline{9})$.

Entre estas últimas se encuentran la necrólisis epidérmica tóxica (NET) asociada al lupus eritematoso cutáneo agudo o subagudo, que se caracteriza por lesiones tipo NET en regiones típicas de lupus cutáneo agudo o subagudo con tendencia a que las lesiones estén en áreas fotodistribuidas; la necrólisis epidérmica tóxica que ocurre en pacientes con lupus eritematoso sistémico sin evidencia de lesiones cutáneas específicas de lupus y el lupus cutáneo subagudo o crónico con vesículas o ampollas (9).

Es importante aclarar que la manifestación tipo NET del lupus también se ha descrito como manifestación tipo síndrome de Stevens Johnson (SSJ) o sobre-posición SSJ/NET, dependiendo del porcentaje de la superficie corporal comprometida así: SSJ, cuando es menor del $10 \%$; SSJ/NET, si está entre 10 y $30 \%$; y, NET, cuando es mayor al $30 \%(\underline{10}, \underline{11})$.

También hay que tener en cuenta que los pacientes con lupus eritematoso cutáneo o sistémico pueden presentar brotes con vesículas y ampollas no asociadas al lupus como dermatitis de contacto ampollosa, herpes simple, etc. (9) 
Esta condición está ampliamente aceptada en la literatura actual porque hay múltiples reportes de caso en los que se descarta la hipersensibilidad medicamentosa o se clasifica como altamente improbable.
La clasificación de las manifestaciones vésico ampollosas del lupus ha sido difícil y controversial. Hay autores que opinan que la injuria tipo NET por lupus eritematoso no hace parte de las manifestaciones específicas de éste y no es debida a la vacuolización extensa de la membrana basal. Mandelcorn et al. afirman que la NET por lupus es una entidad diferente de lesiones cutáneas relacionadas al lupus eritematoso, donde la causa subyacente es un proceso de apoptosis no controlado (12).

Horne et al. cuestionan que la NET por lupus sea una entidad diferente a la NET clásica medicamentosa (13). Es bien sabido que los pacientes con enfermedad del tejido conectivo tienen mayor riesgo de hacer NET medicamentosa y la gran mayoría están expuestos a múltiples medicamentos; por esto creen que NET por lupus no es una entidad diferente (14).

Sin embargo, esta condición está ampliamente aceptada en la literatura actual porque hay múltiples reportes de caso en los que se descarta la hipersensibilidad medicamentosa o se clasifica como altamente improbable, (9). En la paciente descrita no se puede excluir completamente esta posibilidad, ya que las lesiones de NET ocurrieron después del inicio de cloroquina, losartan y metilprednisolona (ella no recibía ningún medicamento previo a esto).

Hay publicaciones que tratan de comparar la clínica e histopatología de la NET clásica medicamentosa con el lupus eritematoso con presentación tipo NET para poder distinguirlos $(15,16)$. Algunos describen que los pacientes con lupus eritematoso con injuria tipo NET presentan una progresión más lenta del cuadro, no tienen relación temporal con el inicio de medicamentos, presentan serología positiva para lupus y los síntomas sistémicos son menores $(\underline{15}, \underline{16})$. También refieren que en ambas entidades hay compromiso mucoso y la histopatología en ambas entidades es igual (necrosis epitelial con infiltrado linfocítico superficial) con inmunofluorescencia negativa (12).

En el 2011, Ziemer et al. (10) realizan un estudio retrospectivo en el cual buscan en el registro nacional alemán los casos de SJS/NET en pacientes con lupus eritematoso sistémico. Encontraron 1366 casos, de los cuales identificaron 17 con historia bien documentada de lupus y material histológico representativo. Estos casos los dividieron según historia clínica en aquellos que representaban SSJ/NET clásica medicamentosa y aquellos que eran más probablemente lupus eritematoso con manifestación tipo NET.

Estos autores proponen algunas características clínicas e histológicas que, aunque no concluyentes por sí mismas, pueden ayudar a diferenciar ambas entidades: la exacerbación del lupus eritematoso sistémico al momento de la aparición de lesiones en piel, la fotodistribución de las lesiones, la presencia de lesiones anulares y la ausencia o leve compromiso erosivo mucoso favorece el diagnóstico de lupus tipo NET sobre NET clásica.

La alteración vacuolar en la unión dermoepidérmica, con la presencia de queratinocitos necróticos en los niveles bajos de la epidermis, el infiltrado linfocítico perivascular con melanófagos y mucina son las características histopatológicas que apuntan a un origen lúpico. Para este caso no se contó con la inmunofluorescencia, ya que era un estudio retrospectivo.

En la paciente descrita, la exacerbación del lupus coincidió con las lesiones en piel; sin embargo, estas no eran foto-distribuidas pero sí anulares y había compromiso 
La existencia del síndrome de Rowell ha sido controvertida. mucoso. Lamentablemente, tampoco se contó con la histopatología, por lo que según los criterios anteriores es difícil de clasificar.

Ting et al. proponen para esta lesión tipo NET no asociada a hipersensibilidad a medicamentos -no sólo descrita como manifestación de lupus eritematoso, sino también de enfermedad de injerto contra huésped-, el nombre de síndrome agudo de panepidermolisis apoptótica (ASAP, por sus sigla en inglés que también se usan para abreviar As Soon As Possible) (9), pero este no ha sido ampliamente adoptado en la literatura.

Torchia et al. proponen no usar los términos "lupus cutáneo agudo" o "subagudo tipo NET" sino "lupus cutáneo agudo" o "subagudo con lesiones tipo NET" ya que, según ellos, no se trata de lupus cutáneo con manifestaciones del NET clásico (11).

Mandelcorn et al., en 2003, reportan dos casos muy similares al descrito, puesto que las lesiones iniciales también fueron tipo eritema multiforme que progresaron a NET. Los autores refieren que, aunque se podría considerar como diagnóstico diferencial el síndrome de Rowell (caracterizado por lesiones tipo EM en pacientes con lupus eritematoso), no se ha descrito la progresión a NET en pacientes con Rowell (12).

Otros autores, en cambio, sugieren que el síndrome de Rowell y la NET por lupus eritematoso hacen parte de un espectro, en el que el síndrome de Rowell puede ser una forma limitada de expresión del lupus tipo NET y en el que el mecanismo patogénico en ambas entidades corresponde al mismo proceso $(\underline{9}, \underline{10})$.

Cabe anotar que la existencia del síndrome de Rowell ha sido controvertida: inicialmente Rowell reporta cuatro pacientes con lupus eritematoso discoide asociado a eritema multiforme, ANA's con patrón moteado y anticuerpos anti extracto salino de tejido humano (aún no está claro si estos anticuerpos representaban anti Ro o anti La), perniosis y factor reumatoide positivo (17).

Sin embargo, se han reportado casos de síndrome de Rowell en pacientes que no cumplían los criterios originales. También, se han publicado reportes de pacientes, no sólo con lupus discoide sino también lupus agudo o subagudo asociados a lesiones tipo eritema multiforme, que muchas veces tampoco tenían la histología o inmunofluorescencia característica $(\underline{17}, \underline{18})$.

Debido a que Rowell describió este síndrome antes de que se definiera el lupus cutáneo subagudo, varios pacientes diagnosticados con este síndrome probablemente tenían dicha condición. Ya que el lupus subagudo se caracteriza por lesiones anulares policíclicas (que pueden ser lesiones en diana tipo eritema multiforme), ANA'S con patrón moteado, anti-Ro y anti-La positivos (la perniosis, a su vez, se relaciona con la presencia de estos auto-anticuerpos). Otra posibilidad es que estos pacientes cursaran con lupus eritematoso cutáneo y eritema multiforme clásico concomitante, lo cual no representa un síndrome característico $(17,18)$.

Torchia et al. consideran que el síndrome de Rowell merece considerarse como una entidad aparte, dada la baja frecuencia con la que el eritema multiforme se asocia a anormalidades inmunológicas (auto anticuerpos anti Ro y anti La) y al lupus; además en estos casos no se encuentra un desencadenante infeccioso, como en la 
En cuanto al tratamiento del lupus tipo NET, hay múltiples reportes de casos tratados con inmunoglobulina humana, similar al tratamiento de la NET clásica. mayoría de los casos de eritema multiforme. Finalmente, la localización de las lesiones en el síndrome de Rowell no es acral o mucosa como en el eritema multiforme clásico, por lo cual incluso han propuesto nuevos criterios (11).

En cuanto al tratamiento del lupus tipo NET, hay múltiples reportes de casos tratados con inmunoglobulina humana, similar al tratamiento de la NET clásica $(\underline{9}, 12, \underline{13}, 19)$. Se cree que el mecanismo de acción de la inmunoglobulina es a través del bloqueo de la interacción Fas-Fas ligando (mediadora de la apoptosis) mediante anticuerpos $(\underline{9}, \underline{12}, \underline{13})$.

En el caso de la paciente reportada, debido a la nefropatía lúpica severa y los efectos que puede tener la inmunoglobulina humana en cuanto a sobrecarga de volumen en pacientes con alteración de la función renal, se decidió no aplicarla. También hay reportes exitosos descritos con plasmaféresis (20).

\section{Conclusión}

El lupus con manifestación tipo NET es un concepto en evolución que aun no está claramente definido, pero que se acepta en la literatura mundial debido a los múltiples reportes de casos. Las manifestaciones tipo eritema multiforme-like en el lupus y el síndrome de Rowell también son entidades controvertidas.

\section{Bibliografía}

1. Walling HW, Sontheimer RD. Cutaneous lupus erythematosus: issues in diagnosis and treatment. Am. J. Clin. Dermatol. 2009;10(6):365-81.

2. Obermoser G, Sontheimer R, Zelger B. Overview of common, rare and atypical manifestations of cutaneous lupus erythematosus and histopathological correlates. Lupus. 2010;19(9):1050-70.

3. Tincopa M, Puttgen KB, Sule S, Cohen BA, Gerstenblith MR. Bullous lupus: an unusual initial presentation of systemic lupus erythematosus in an adolescent girl. Pediatr. Dermatol. 2010;27(4):373-6.

4. Gammon WR, Briggaman RA. Bullous SLE: a phenotypically distinctive but immunologically heterogeneous bullous disorder. J. Invest. Dermatol. 1993;100(1):28S-34S.

5. Yung A, Oakley A. Bullous systemic lupus erythematosus. Australas. J. Dermatol. 2000;41(4):234-7.

6. Sebaratnam DF, Murrell DF. Bullous Systemic Lupus Erythematosus. Dermatol. Clin. 2011;29(4):649-53.

7. Fleming MG, Bergfeld WF, Tomecki KJ, Tuthill RJ, Norris M, Benedetto EA, et al. Bullous systemic lupus erythematosus. Int. J. Dermatol. 1989;28(5):321-6.

8. Kaplan RP, Callen JP. Overlapping cutaneous disorders related to dermatitis herpetiformis. Clin. Dermatol. 1991;9(3):361-8.

9. Ting W, Stone M, Racila D, Scofield R, Sontheimer R. Toxic epidermal necrolysis-like acute cutaneous lupus erythematosus and the spectrum of the acute syndrome of apoptotic pan-epidermolysis (ASAP): a case report, concept review and proposal 
for new classification of lupus erythematosus vesiculobullous skin lesions. Lupus. 2004;13(12):941-50.

10. Ziemer M, Kardaun SH, Liss Y, Mockenhaupt M. Stevens-Johnson syndrome and toxic epidermal necrolysis in patients with lupus erythematosus: a descriptive study of 17 cases from a national registry and review of the literature. Br. J. Dermatol. 2012;166(3):575-600.

11. Torchia D, Romanelli P, Kerdel FA. Erythema multiforme and Stevens-Johnson syndrome/toxic epidermal necrolysis associated with lupus erythematosus. J. Am. Acad. Dermatol. 2012;67(3):417-21.

12. Mandelcorn R, Shear NH. Lupus-associated toxic epidermal necrolysis: a novel manifestation of lupus? J. Am. Acad. Dermatol. 2003;48(4):525-9.

13. Horne NS, Narayan AR, Young R-M, Frieri M. Toxic epidermal necrolysis in systemic lupus erythematosus. Autoimmun. Rev. 2006;5(2):160-4.

14. Cisneros CGC, Romiti R, Santi CG, Aoki V, Valente NYS, Nico MMS. Toxic epidermal necrolysis-like cutaneous lupus erythematosus: A series of three patients. Acta Derm. Venereol. 2010;90(2):175-8.

15. Boontaveeyuwat E, Silpa-archa N, Kulthanan K. Toxic epidermal necrolysis-like acute cutaneous lupus erythematosus (TEN-like ACLE) in SLE patients: a report of two cases. Asian Pac. J. Allergy Immunol. Launched Allergy Immunol. Soc. Thail. 2012;30(1):83-7.

16. Paradela S, Martínez-Gómez W, Fernández-Jorge B, Castiñeiras I, Yebra-Pimentel T, Llinares $P$, et al. Toxic epidermal necrolysis-like acute cutaneous lupus erythematosus. Lupus. 2007;16(9):741-5.

17. Shteyngarts AR, Warner MR, Camisa C. Lupus erythematosus associated with erythema multiforme: does Rowell's syndrome exist? J. Am. Acad. Dermatol. 1999:40(5 Pt 1):773-7.

18. Antiga E, Caproni M, Bonciani D, Bonciolini V, Fabbri P. The last word on the socalled «Rowell»s syndrome'? Lupus. 2012;21(6):577-85.

19. Torchia D, Caproni M, Massi D, Chella A, Fabbri P. Paraneoplastic toxic epidermal necrolysis-like subacute cutaneous lupus erythematosus. Clin. Exp. Dermatol. 2010;35(4):455-6.

20. Simsek I, Cinar M, Erdem H, Pay S, Meric C, Dinc A. Efficacy of plasmapheresis in the treatment of refractory toxic epidermal necrolysis-like acute cutaneous lupus erythematosus. Lupus. 2008;1 7(6):605-6. 